\title{
The K296-D320 region of recombinant levansucrase BA-SacB can affect the sensitivity of Escherichia coli host to sucrose
}

\author{
Song Gao ${ }^{1} \cdot$ Bo Jiang ${ }^{2} \cdot$ Song Zhu ${ }^{2} \cdot$ Darren J. Hart ${ }^{3} \cdot$ Xia Liu ${ }^{1} \cdot$ Hongling Wang ${ }^{1,4} \cdot$ Yingfeng An $^{1}$
}

Received: 22 February 2019 / Accepted: 7 July 2019/Published online: 22 July 2019

(C) Università degli studi di Milano 2019

\begin{abstract}
Purpose A mutant BA-SacB-Del encoding BA-SacB minus K296-D320 region was constructed to analyze its effect on catalytic characteristics of the enzyme as well as help deepen understanding of the catalytic mechanism of BA-SacB and even proteins of GH68 family.

Methods Based on the comparison of levansucrases from Bacillus amyloliquefaciens (BA-SacB) and Sphingopyxis macrogoltabida (SM-Lev), a mutant BA-SacB-Del encoding BA-SacB minus K296-D320 region was constructed and its effect on catalytic characteristics of the enzyme was analyzed.

Results Deletion of this region would undoubtedly affect the conserved structure (i.e., central negatively charged cavity surrounded by five antiparallel $\beta$-strands) shared by the GH68 family. Therefore, Escherichia coli-expressing mutant BASacB-Del could more efficiently catalyze the production of levan in media containing high concentration of sucrose, which is unrealizable for BA-SacB.

Conclusions This result should be valuable for understanding this conditional lethal mechanism. Therefore, this study should be very valuable for understanding the catalytic mechanism of BA-SacB and even proteins of the GH68 family. More importantly, levan can be conveniently produced by direct fermentation of sucrose-containing media with E. coli-expressing BA-SacB-Del which is not sensitive to sucrose.
\end{abstract}

Keywords Bioinformatics analyses $\cdot$ Fructosyltransferase $\cdot$ Levansucrase $\cdot$ Molecular evolution $\cdot$ Recombinantprotein expression

\section{Introduction}

Levansucrases (EC 2.4.1.10) are enzymes catalyzing sucrose hydrolysis by hydrolase activity and levan synthesis by fructosyltransferase activity. Levan has been widely used for

Electronic supplementary material The online version of this article (https://doi.org/10.1007/s13213-019-01496-1) contains supplementary material, which is available to authorized users.

Yingfeng An

anyingfeng666@163.com

1 College of Biosciences and Biotechnology, Shenyang Agricultural University, No.120 Dongling Road, Shenyang 110161, People's Republic of China

2 State Key Laboratory of Food Science and Technology, Jiangnan University, Wuxi 214122, China

3 Institut de Biologie Structurale (IBS), CEA, CNRS, University Grenoble Alpes, 38044 Grenoble, France

4 College of Food Science, Shenyang Agricultural University, Shenyang, China personal care, medical applications, prebiotics, aquaculture, films of packaging, foods, etc. (Öner et al. 2016). Therefore, increasing attention has recently been focused on biosynthesis of levan and Fructooligosaccharides (FOSs) by microbial levansucrase. Currently, levansucrases have been identified from a wide range of microorganisms belonging to the genera Acetobacter, Bacillus, Erwinia, Geobacillus, Lactobacillus, Leuconostoc, Pseudomonas, Zymomonas, etc. (Li et al. 2015). According to the database of carbohydrate-active enzymes (CAZY) (Henrissat and Davies 1997), levansucrases belong to family 68 of the glycoside hydrolases (GH68). All the GH68 enzymes use sucrose as their preferential donor substrate. The levansucrases could realize transfructosylation through a ping-pong mechanism involving the formation of a transient fructosyl-enzyme intermediate (Hernandez et al. 1995). At high sucrose concentrations levansucrase could transfer fructosyl units to various acceptors including fructan, glucose and sucrose, whereas at low sucrose concentrations it functions mainly as a hydrolase with water as acceptor. Commonly levansucrases from bacteria could catalyze all these reactions but with different efficiency (Öner et al. 
2016). The members of GH68 share a $\beta$-propeller fold consisting of a central negatively charged cavity and five antiparallel $\beta$-strands (Strube et al. 2011). Until now, structures of some levansucrases have been solved, such as the ones from B. subtilis (PDB ID: 1OYG) (Manival et al. 1997), Gluconacetobacter diazotrophicus SRT4 (PDB ID: 1W18) (Martínez-Fleites et al. 2005), B. megaterium (PDB ID: 3OM2) (Strube et al. 2011), E. amylovora (PDB ID: 4D47) (Wuerges et al. 2015), and E. tasmaniensis (PDB ID: 6FRW) (Polsinelli et al. 2019). All these structures show the basic features of enzymes from the GH68 family. In addition, the effect of some important amino residues on the structure and function of levansucrases have been studied (Chambert and Petit-Glatron 1991; Ozimek et al. 2004; Ozimek et al. 2006; Strube et al. 2011). However, deep understanding of the catalytic mechanisms of these enzymes is still necessary.

According to the multiple alignments of deduced amino acid sequences, all bacterial levansucrases share seven conserved regions. However, many amino acid residues from Gram-positive and Gram-negative origin are obviously different (Arrieta et al. 1996; Seo et al. 2000). It is worth noting that the pairwise alignments between sequences of levansucrases from Gram-positive and Gram-negative bacteria show some alignment gaps. Whether the sequence of these alignment gaps could affect the characteristics of levansucrases deserves further study. Additionally, the sequence of these alignment gaps might be valuable for understanding molecular mechanism of levansucrases and provide evolution-related information of these enzymes. Molecular evolution of protein can provide an important means of studying the genetic relationship between protein molecules. It is a valuable tool for analyzing evolutionary relationships of protein molecules through comparing their sequences. According to the alignment of protein sequences, the regions with the same amino acids are commonly considered conservative regions which might share common ancestors. Whereas the gaps identified during pairwise alignments show that deletion, insertion, or replacement that might have taken place in these regions during the evolutionary process. Therefore, analysis of these alignment gaps would help us to understand the relationship between protein structures and functions, as well as the evolutionary process of the proteins (Chowdhury and Garai 2017). Moreover, the commonly used methods for modifying DNA molecules include random mutagenesis, site-directed mutagenesis, and DNA recombination, while introducing mutations through modifying alignment gaps has rarely been reported (Packer and Liu 2015). When protein sequence of levansucrase from Bacillus amyloliquefaciens (BA-SacB) (GenBank no AFJ64285.1) was searched using BLAST against all proteins in GenBank, the protein with minimum sequence identity to BA-SacB was levansucrase from Sphingopyxis macrogoltabida (SM-Lev). According to pairwise alignment of protein sequences of BA-SacB and
SM-Lev, the region of K296-D320 is missing in SM-LEV as compared with BA-SacB. This region should be very important because it contains one $\beta$-strand of the conserved "five antiparallel $\beta$-strands," but it might be lost during evolution of levansucrase. Until now, how can this region affect the characteristics of levansucrases has not been reported. It is well known that the expression of SacB in the presence of sucrose is toxic to various Gram-negative bacteria including E. coli (Cai and Wolk 1990; Jäger et al. 1992; Jäger et al. 1995; Pelicic et al. 1996). Therefore, convenient production of valuable levan by direct fermentation of sucrose containing media with $E$. coli-expressing $\mathrm{SacB}$ is usually impractical.

In this study, a mutant BA-SacB-Del encoding BA-SacB minus the K296-D320 region was constructed and its effect on catalytic characteristics of enzyme was analyzed. In addition, sucrose sensitivity of BA-SacB-Del-expressing E. coli strain was detected to see the possibility of producing levan by direct fermentation of sucrose-containing media.

\section{Materials and methods}

\section{Cloning of BA-SacB gene and BA-SacB-Del mutant encoding BA-SacB minus K296-D320 region}

Genomic DNA was extracted from B. amyloliquefaciens and used as a template for PCRs. BA-SacB gene was amplified by PCR using primers: BA-SacB-For (5'-CTAGG ACGTC GATGA ACATC AAAAA ATTTG CAAA $\overline{\text { CGAGC }}$ CAC-3', Aat II underlined) and BA-SacB-Rev (5'-TACCA CTAGT GTTGT TAACC GTAAG CTGTC CTTGT TCAAG G-3', Spe I underlined). The PCR product was cloned into the corresponding restriction enzyme sites of pETM11, to give plasmid pETM-BA-SacB containing BASacB gene. Then, BA-SacB-Del mutant encoding BA-SacB minus K296-D320 region was introduced by overlap extension PCR using BA-SacB-For and BA-SacB-Rev as external primers, and Inner-For (5'-CGAAG CCAAC ACTGG AACAG ATAAC AGTGC TAAAA AACGC GATGC TGAAT TAG-3') and Inner-Rev (5'-CTAAT TCAGC ATCGC GTTTT TTAGC ACTGT TATCT GTTCC AGTGT TGGCT TCG-3') as inner primers. The BA-SacBDel mutant was cloned into pETM11 to give plasmid pETMBA-SacB-Del.

\section{Analysis of the sucrose sensitivity of $E$. coli BL21(DE3) /pETM-BA-SacB-Del strain and its fermentation products}

The plasmids pETM-BA-SacB-Del, pETM-BA-SacB, and pETM11 were transformed into E. coli BL21(DE3) to give strains $E$. coli BL21(DE3)/pETM-BA-SacB-Del, E. coli BL21(DE3)/pETM-BA-SacB, and E. coli BL21(DE3)/ 
pETM11, respectively. To assay sucrose sensitivity of $E$. coli BL21(DE3)/pETM-BA-SacB-Del strain, these strains were cultured on LB agar plate and then single colonies were spotted on LB agar plates supplemented with $0.1 \mathrm{mmol} / \mathrm{L}$ isopropyl- $\beta$-d-thiogalactopyranoside (IPTG), $50 \mathrm{mg} / \mathrm{L}$ kanamycin, and 5\% sucrose. The plate was incubated at $25{ }^{\circ} \mathrm{C}$ for $72 \mathrm{~h}$. Then, totally, $0.1 \mathrm{~g}$ cells were scraped from the colonies of E. coli BL21(DE3)/pETMBA-SacB-Del and resuspended in $5 \mathrm{~mL}$ water. After centrifugation $(13,000 \mathrm{~g}, 10 \mathrm{~min})$, the supernatant was recovered and the components of reducing sugars were detected using the method of 2,4-dinitrosalicyclic acid (DNS) assay (Bailey 1988), and 5\% sucrose was used as a control during the assay process. Then, these components were further analyzed by high-performance liquid chromatography (HPLC) on a Shodex Ionpack KS-802 column (Showa Denko, Tokyo). Fructofuranosyl-nystose (GF4), nystose (GF3), 1-kestose (GF2), sucrose (GF), glucose (G), and fructose (F) (Meiji Seika Kaisha Ltd.) were used as standards. The eluate was monitored using a RI detector, and the mobile phase was water at $0.5 \mathrm{~mL} / \mathrm{min}$. The temperature of the column and detector was $25{ }^{\circ} \mathrm{C}$. E. coli BL21(DE3)/pETM-BA-SacB-Del and BL21(DE3)/ pETM-BA-SacB were cultured in LB media and protein expression was induced by $0.1 \mathrm{mmol} / \mathrm{L}$ IPTG. The same amount of cells of the two strains were pelleted by centrifugation, resuspended in sodium phosphate buffer ( $50 \mathrm{mmol} / \mathrm{L}, \mathrm{pH} 5.9$ ), and lysed by 5 cycles of sonication (30 s, $50 \%$ cycle, $65 \%$ power) with an interval of $5 \mathrm{~min}$ between each cycle. The expressed BA-SacB-Del and BASacB proteins were purified using Ni-NTA agarose beads (Qiagen). Then, catalytic activities of BA-SacB-Del and BA-SacB were analyzed using crude enzyme or purified enzymes in presence of 5\% sucrose. The reactions were incubated at $40{ }^{\circ} \mathrm{C}$ for $5 \mathrm{~h}$ and the production of glucose was estimated using Glucose Assay Kit (HUILI Biotech Co., China). Enzyme activity assays were performed in triplicate. E. coli BL21(DE3)/pETM-BA-SacB-Del was used to inoculate a TB medium supplemented with $5 \%$ sucrose and $100 \mu \mathrm{g} / \mathrm{mL}$ kanamycin, and fermentations were performed under static conditions at $25{ }^{\circ} \mathrm{C}$ for $72 \mathrm{~h}$ to produce polysaccharide. The catalysis with purified BASacB was performed under the same conditions to compare with the direct fermentation of $E$. coli BL21(DE3)/pETMBA-SacB-Del for time dependent yields of levan. After centrifugation $(4,000 \mathrm{~g}, 5 \mathrm{~min})$, the supernatant of each reaction was recovered and mixed with four volumes of ethanol. After centrifugation at $13,000 \mathrm{~g}$ for $10 \mathrm{~min}$, the pellets were washed five times with $50 \%$ ethanol and dehydrated by lyophilization. Then the linkage type of the pellet of polysaccharide produced by direct fermentation of $E$. coli BL21(DE3)/pETM-BA-SacB-Del was analyzed using ${ }^{13} \mathrm{C}$ NMR spectrometry. ${ }^{13} \mathrm{C}$ NMR spectrometry was run at
$500 \mathrm{MHz}$ on AMX-500 (Bruker, Germany). In all the assays above, E. coli BL21(DE3)/pETM-BA-SacB and $E$. coli BL21(DE3)/pETM11 were used as a positive and negative control, respectively.

\section{Results and discussion}

In order to investigate the effect of the amino acids K296D320 on the characteristics of BA-SacB, we deleted the DNA sequence encoding K296-D320 from BA-SacB to give BA-SacB-Del. The strains E. coli BL21(DE3)/pETM-BASacB-Del and E. coli BL21(DE3)/pETM-BA-SacB were cultured on LB plate supplemented with IPTG and 5\% sucrose. As a result, E. coli BL21(DE3)/pETM-BA-SacB could not grow on the plate, while E. coli BL21(DE3)/pETM-BASacB-Del could form colonies (Fig. 1a). This result indicated that deletion of the region K296-D320 of BA-SacB made the $E$. coli host be less or even not sensitive to sucrose. Interestingly, the colonies formed by E. coli BL21(DE3)/ pETM-BA-SacB-Del were mucoid, which are obviously different from that formed by E. coli BL21(DE3)/pETM11. Then, these mucoid colonies were scraped from the plate, resuspended in water, and DNS assay was performed DNS assay on the supernatant after centrifugation (Fig. 1b). The positive result indicated that BA-SacB-Del could introduce the production of reducing sugars on LB agar plate. Then the reducing sugars were identified as glucose and fructose
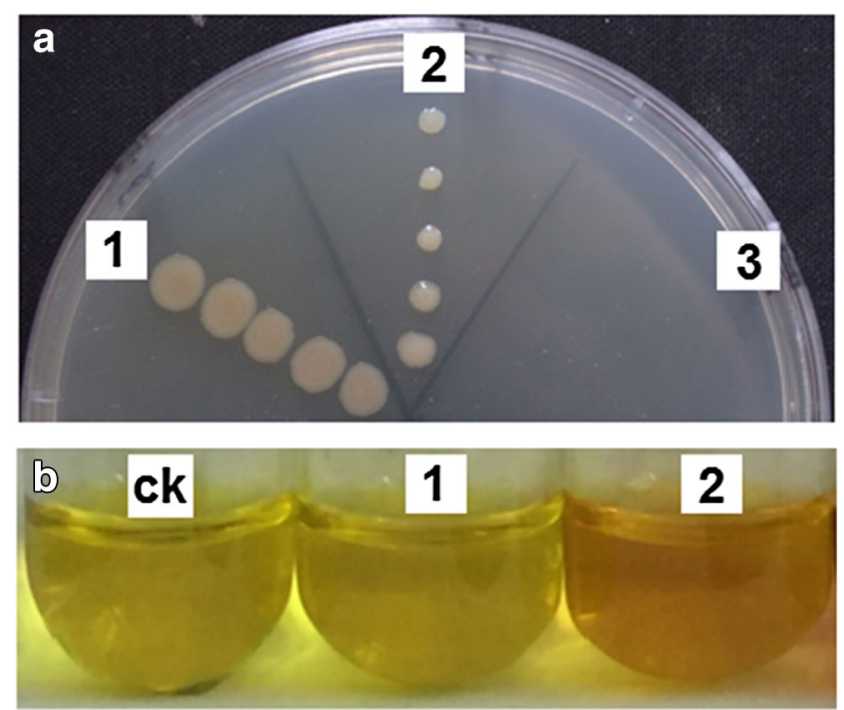

Fig. 1 The change of colony morphology of E. coli and the production of reducing sugars introduced by BA-SacB-Del in the presence of sucrose. a Shows the culture of $E$. coli BL21(DE3) harboring different plasmids on LB agar plate supplemented with IPTG and sucrose at $25^{\circ} \mathrm{C}$ for $72 \mathrm{~h}$. bSshows the DNS assays of reducing sugars produced by colonies scraped from agar plate. 1, 2, and 3 refer to colonies or DNS assays be associated with E. coli BL21(DE3) harboring plasmids pETM11, pETMBA-SacB-Del, and pETM-BA-SacB, respectively. CK refers to DNS reaction only in the presence of $5 \%$ sucrose 
according to the analysis of HPLC (Fig. 2), indicating that the mutant BA-SacB-Del has strong sucrose hydrolase activity. This result could account for formation of the mucoid colonies because fructose is very hygroscopic. In addition, nearly no fructooligosaccharides could be detected, indicating that BASacB-Del has high product specificity. Fermentation of sucrose-containing media with $E$. coli BL21(DE3)/pETMBA-SacB-Del can produce turbid polysaccharide (Fig. 3a, b). Then structure of the polysaccharide was analyzed by ${ }^{13} \mathrm{C}$ NMR spectrometry. Assignment of the peaks was based on the report of Shimamura et al (1987). The results confirmed that the polysaccharide was levan of $\beta$-2,6-fructan (Fig. 4). It is worth noting that only linkage type of the insoluble polymer with high molecular mass has been analyzed by ${ }^{13} \mathrm{C}$ NMR spectrometry, because the soluble sucrose, glucose, and fructose in the reaction mixture have been eliminated by repeated washing with $50 \%$ ethanol. Levan can be conveniently produced by direct fermentation of sucrose containing media with E. coli-expressing BA-SacB-Del which is not sensitive to sucrose. By contrast, currently the commonly used strategy to produce levan with recombinant $E$. coli strains was in vitro catalysis using isolated levansucrase (Xu et al. 2017; Gao et al. 2017a; Porras-Domínguez et al. 2017; Xu et al. 2018; Núñez-López et al. 2019; Polsinelli et al. 2019; Szwengiel and Wiesner 2019), which is less convenient than
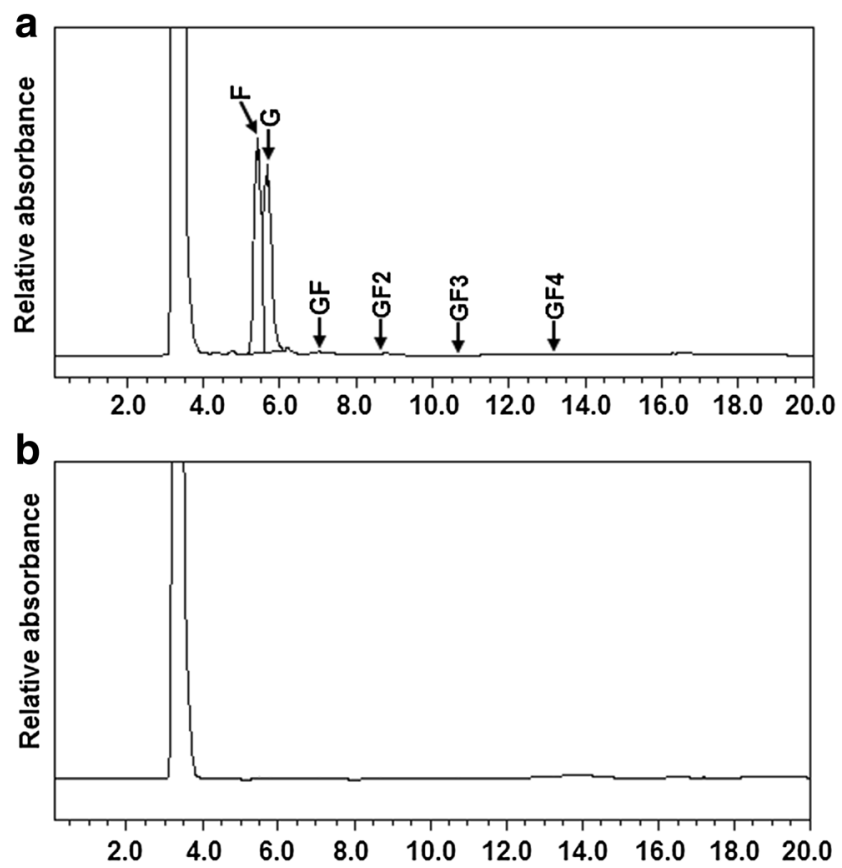

Fig. 2 HPLC chromatogram shows analysis of the substances introducing the formation of mucoid colonies by E. coli BL21(DE3)/ pETM-BA-SacB-Del. a Shows the component of the solution after washing colonies formed by E. coli BL21(DE3)/pETM-BA-SacB-Del. b shows the component of the solution after washing colonies formed by $E$. coli BL21(DE3)/pETM11. F, G, GF, GF2, GF3, and GF4 refer to predicted locations of fructose, glucose, sucrose, 1-kestose, nystose, and fructofuranosyl-nystose, respectively
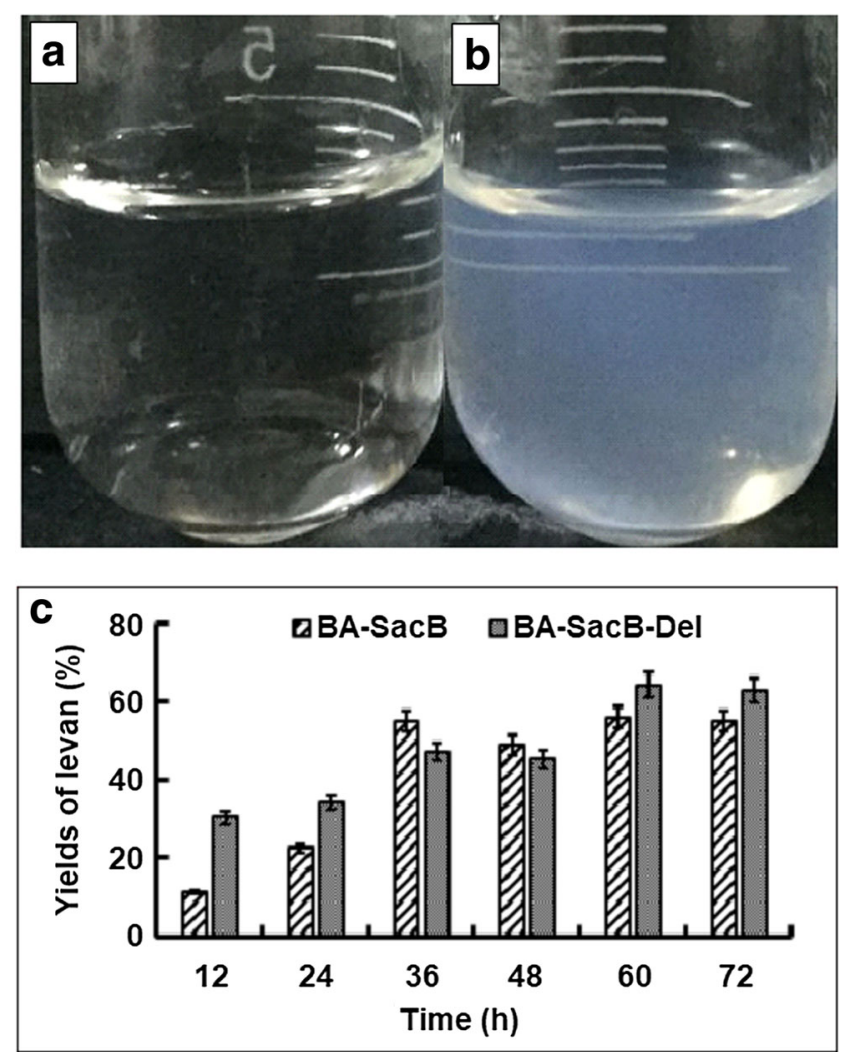

Fig. 3 Production of levan by direct fermentation with E. coli-expressing BA-SacB-Del or catalysis with purified BA-SacB. a and $\mathbf{b}$ refer to reactions associated with direct fermentation of sucrose-containing media with $E$. coli BL21(DE3)/pETM11 and E. coli BL21(DE3)/ pETM-BA-SacB-Del, respectively. c shows comparison of catalysis with purified $\mathrm{BA}-\mathrm{SacB}$ and direct fermentation with $E$. coli BL21(DE3)/pETM-BA-SacB-Del for time-dependent yields of levan

direct fermentation because the steps of protein purification are always required. In addition, during direct fermentation the by-product of glucose could provide nutrition for growing $E$. coli, thus making it possible to further reduce the cost of the culture medium. The direct fermentation of E. coli

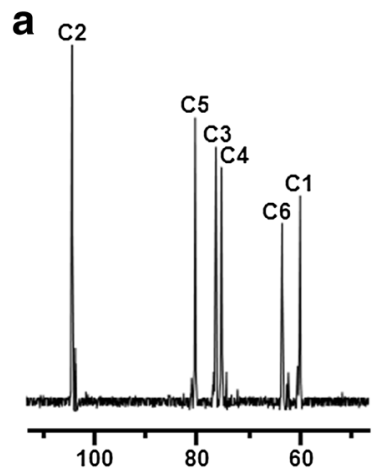

b

Fig. $4{ }^{13} \mathrm{C}$-NMR spectra used for the analysis of polysaccharide produced by fermentation of sucrose-containing media with $E$. coli BL21(DE3)/pETM-BA-SacB-De1. a ${ }^{13} \mathrm{C}-\mathrm{NMR}$ spectra shows polysaccharide catalyzed using BA-SacB-Del. b Shows chemical shifts for ${ }^{13} \mathrm{C}-\mathrm{NMR}$ spectra of levan and polysaccharide synthesized using BASacB-Del 
BL21(DE3)/pETM-BA-SacB-Del was compared with catalysis using purified BA-SacB for time-dependent yields of levan. As a result, although direct fermentation of $E$. coli BL21(DE3)/pETM-BA-SacB-Del could accumulate levan faster in the early stage, both methods could introduce about $60 \%$ yields of levan after $72 \mathrm{~h}$ of reaction (Fig. 3c). Therefore, direct fermentation of sucrose-containing media with $E$. coliexpressing BA-SacB-Del provided a valuable option for convenient and efficient production of levan.

It is well known that insertional inactivation of a plasmidborne SacB expression cassette can be used for selection of bacterial clones containing recombinant DNA inserts. Therefore, SacB genes have been widely used for marker exchange procedures to trap insertion sequences (Thulin et al. 2015; Sawisit et al. 2015; Rubio et al. 2015; Weissman 2016). There are two hypotheses to explain the lethality of SacB in the presence of sucrose: (i) production of toxic metabolites by transfer of fructose residues to inappropriate acceptor molecules (Pelicic et al. 1996; Dziewit and Bartosik
2015; Khetrapal et al. 2015), and (ii) accumulation of levan in the periplasm might disrupt periplasmic functions (Jäger et al. 1992; Pelicic et al. 1996). Recently, in our laboratory, we have proven that signal peptide-dependent protein translocation pathway is crucial for the sucrose sensitivity of SacBexpressing $E$. coli, indicating that accumulation of levan in the periplasm should account for this phenomenon (Gao et al. 2017b). However, in this study, although BA-SacB-Del contained signal peptide and had levansucrase activity, E. coli BL21 (DE3)-expressing BA-SacB-Del lost sensitivity towards sucrose. This result indicated that the reason for sucrose sensitivity of SacB-expressing E. coli should be complicated, which might be related to the specific protein structure of $\mathrm{SacB}$. The reason for the loss of sucrose sensitivity of BASacB after deletion of the K296-D320 region deserves further investigation.

BA-SacB and SM-LEV sequence alignment based on structural superimposition was generated by ESPript 3.0 (Fig. 5). Secondary-structural elements were labeled using
Fig. 5 BA-SacB and SM-LEV sequence alignment based on structural superimposition. Secondary-structural elements are indicated by squiggles ( $\alpha$-helices) and arrows ( $\beta$-strands). $\alpha$-helices (labeled $\alpha$ ) and $\beta$-strands (labeled $\beta)$ are numbered consecutively. Regions considered important for the activity are underlined and numbered from I to VII. The catalytic triad at the center of the active site is marked with small triangles. The K296-D320 region deleted in this study is boxed
BA-SacB SM-LEV

BA-SacB

SM-LEV

BA-SacB SM-LEV

BA-SacB SM-LEV

BA-SacB SM-LEV

BA-SacB SM-LEV

BA-SacB SM-LEV

BA-SacB SM-LEV

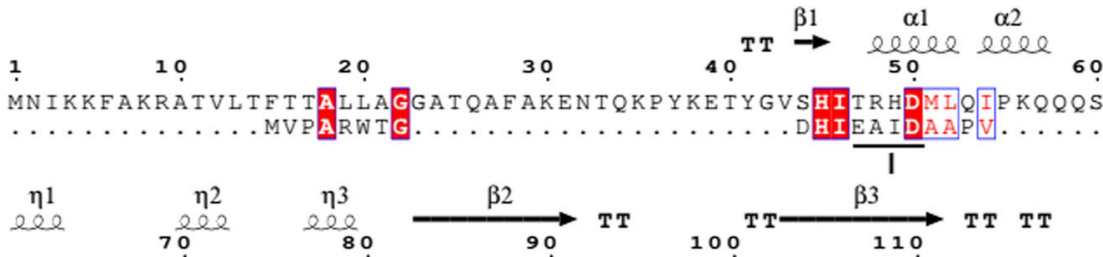

EKYQVPQFDQST I KNIESAKGLDVWDSMPLQNADGTVAEYNGYHVVFA LAGSPKDAD. .

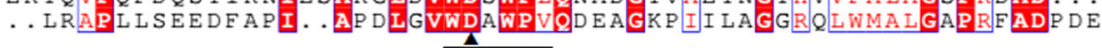
II

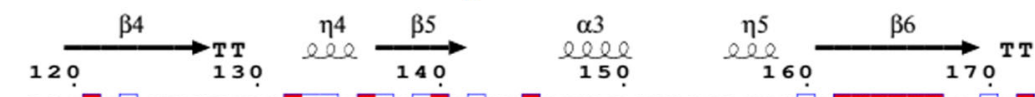
... DTSIYMFYQKVGDNSIDSWKNAGRVFKDSDKFDANDEILKEQTQENSGSATFTSD.G

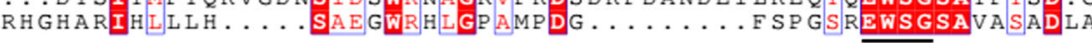

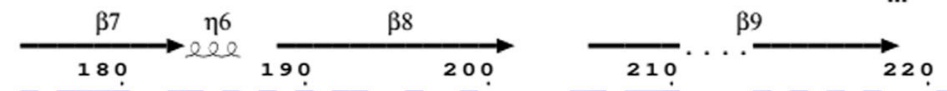

le

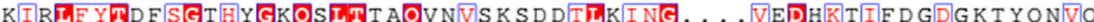
TVALYFTA. TGRE. GEQI LTF EQ RLF S A L A I L L S GEA P R L GDWREL. . E E I V P I D L E
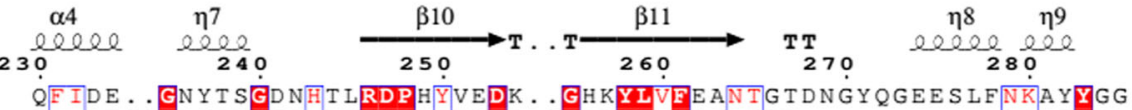
HYMST CGGPSEVGK IKAFRDPAEFRDPAGGDDYLLFAGSOAR ........... SRSEYNG
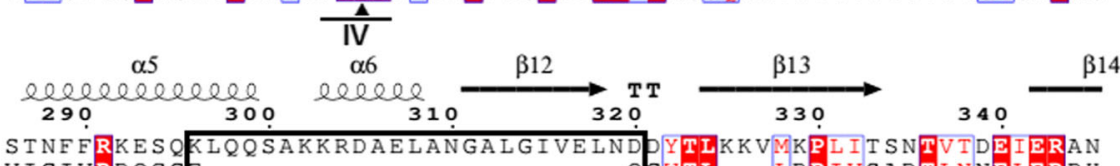
STNF FRKES KLQQSAKKRDAELANGA LGIVELNDPTLKKVMKPLIT SNTVTDEIERAN

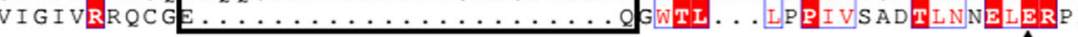

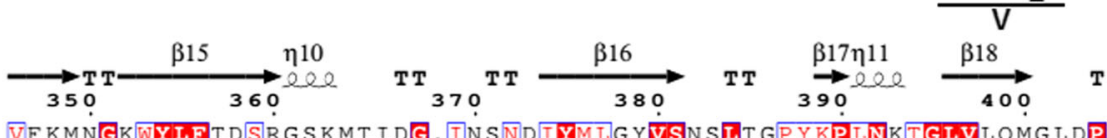

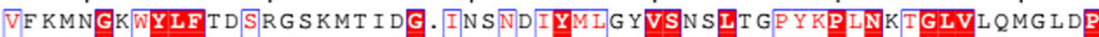
I IFHAGLYYLFWSIQRHVFAP SGP A GPI IYAM. . VSPALFEGWRPVNGSGLVFA... NP VI

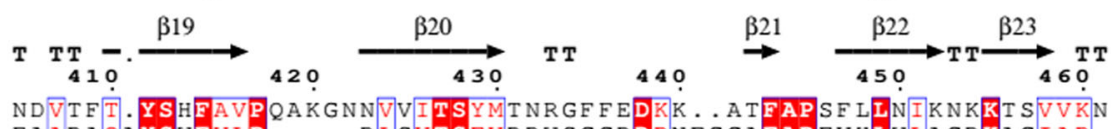

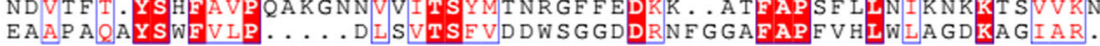


the structure of $B$. subtilis levansucrase as template. BA-sacB presents an overall sequence identity with SM-LEV of $23 \%$. There are totally seven conserved region (Arrieta et al. 1996) and three crucial amino acid residues that function together at the center of the active site (i.e., catalytic triad) in reported levansucrases (Verhaest et al. 2005), but none of them are in the region of K296-D320. To further understand the effect of deleting the K296-D320 region on protein structure and functions, the protein models of BA-SacB and BA-SacB-Del were constructed using SWISS-MODEL (Fig. 6a, b). According to the models, both BA-SacB and BA-SacB-Del have the typical structure of the GH68 family (i.e., $\beta$-propeller fold consisting of five blades with antiparallel $\beta$-strands, and the $\beta$-propeller of each structure forms a central cavity, which is essential for activities). However, all the five blades in BA-SacB comprise four antiparallel $\beta$-strands, while one of the blades in BASacB-Del (Blade III) remains only two antiparallel $\beta$-strands after deletion of the K296-D320 region. According to the structural framework of B. subtilis levansucrase BS-SacB, some important functional motifs in the GH68 family locate in the five blades and form central cavity of the propeller (Meng and Fütterer 2003). The Asp86 and Glue342 form the pair of essential catalytic side chains, whereas Asp247 forms strong hydrogen bonding interactions with hydroxyls of the fructosyl unit of the substrate. Enzyme-substrate hydrogen bonding interactions can promote catalysis by stabilizing the oxocarbenium ion-like transition state of the anomeric carbon. Glu342 is part of a complex network of interactions that includes Arg 246, Arg360, and Tyr411 as well as Glu340 and Glu262. Some of these amino acid residues may also have multiple hydrogen bonding contacts with the substrate (Meng and Fütterer 2003; Martínez-Fleites et al. 2005; Strube et al. 2011). The detailed space distribution and interactions of these amino acid chains were analyzed (Fig. 6c, d). For both BASacB and BA-SacB-Del, these amino acid chains are spaced 3.0-7.5 $\AA$ from each other. After, pairwise comparison of BASacB and BA-SacB-Del, only less than $0.6 \AA$ differences are identified between these amino acids. Therefore, deletion of the K296-D320 region might have not remarkably affected the spatial arrangement of the critical amino acids, which may partly account for the fact that BA-SacB-Del maintains both sucrose hydrolase and fructosyltransferase activities.

Similarities in functions and protein sequences of BA-SacB and SM-Lev suggest that these proteins may originate from same ancestral protein. An interesting question is that: was the K296-D320 region removed from the ancestral protein, or obtained by the ancestral protein during molecular evolution? To assess evolutionary characteristics of levansucrases, pro-
Fig. 6 Protein models of BASacB and BA-SacB-Del. $\mathbf{a}$ and $\mathbf{b}$ show models of BA-SacB and BA-SacB-Del, respectively. The region of K296-D320 is marked in black. $\mathbf{c}$ and $\mathbf{d}$ Show interatomic distances between critical residues in the active sites of BA-SacB and BA-SacB-Del, respectively. Hydrogen bonds are shown by solid lines in green; distances are given in $\AA$
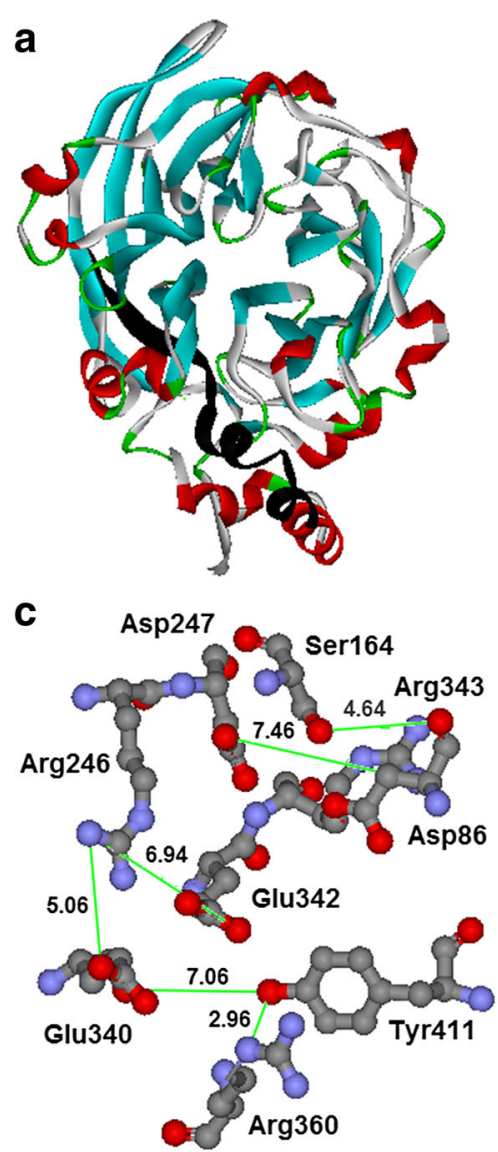
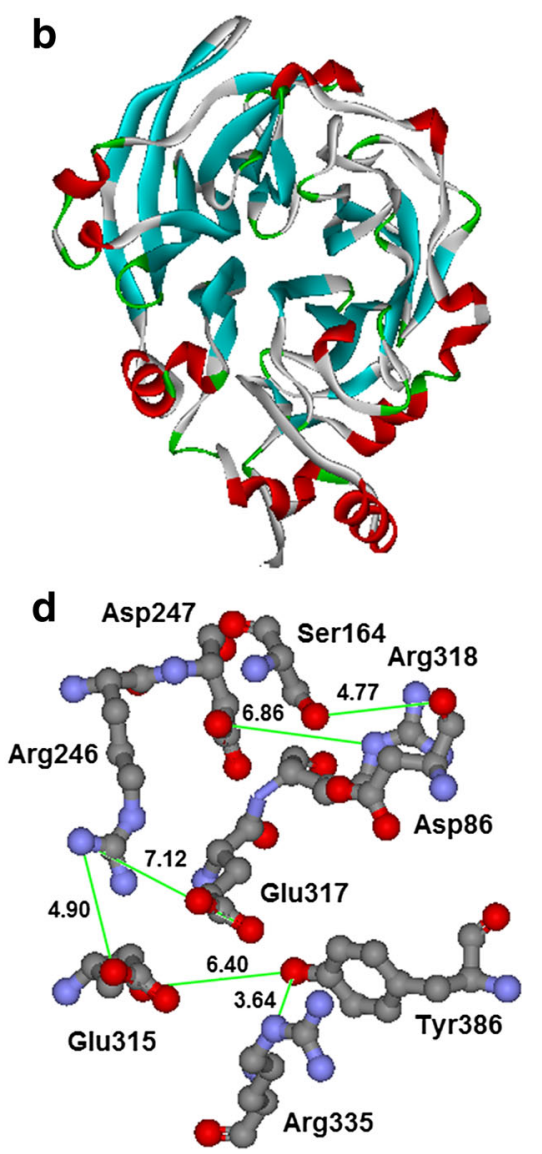
tein sequences of levansucrases having similarity with that of BA-SacB were searched using BLAST against all proteins in GenBank. These levansucrases were found to exist in microorganisms of up to 77 genera. All the characterized levansucrases from each genera (including $B$. amyloliquefaciens and S. macrogoltabida) were performed phylogenetic analysis, and a dendrogram was generated using the MEGA software (Supplementary Fig. 1). According to this dendrogram, levansucrases from nearly all the Grampositive bacteria from different genera (22/24) contain the K296-D320 region. On the other hand, this region is lost or incomplete in all the levansucrases from archaea (i.e., Halogeometricum pallidum, Halogranum salarium, and Halalkalicoccus paucihalophilus) according to this dendrogram. Analysis of evolutionary principle revealed that archaea are more closely related to eukaryotes than to eubacteria (Iwabe et al. 1989; Zaremba-Niedzwiedzka et al. 2017). Therefore, the K296-D320 region might originally exist in the ancestral levansucrase, which might be lost during the evolution of the levansucrases from Gram-negative eubacteria into archaea.

\section{Conclusions}

To sum up, in this study, after comparison and analysis of BASacB and SM-LEV, a mutant BA-SacB-Del encoding BASacB minus K296-D320 region was constructed. The effect of this deleted region on characteristics of the BA-SacB was investigated. This result should be very valuable for understanding sucrose sensitivity of SacB-expressing E. coli. In addition, levan can be conveniently produced by direct fermentation of sucrose-containing media with $E$. coli-expressing BA-SacB-Del which is not sensitive to sucrose. Therefore, this study should be valuable for understanding the mechanisms of BA-SacB and even enzymes of GH68, and lay the foundation for more convenient and efficient production of levan.

Funding information This work was supported by the National Natural Science Foundations of China (grant numbers 31870784, 31100045, 31270114), Program for Liaoning Excellent Talents in University (grant number LR2014018), and Liaoning BaiQianWan Talents Program (grant number 2015-40).

\section{Compliance with ethical standards}

Conflict of interest The authors declare that they have no conflict of interest.

Research involving human participants and/or animals This article does not contain any studies with human participants or animals performed by any of the authors.
Informed consent Informed consent was obtained from all individual participants included in the study.

\section{References}

Arrieta J, Hernández L, Coego A, Suárez V, Balmori E, Menéndez C, Petit-Glatron MF, Chambert R, Selman-Housein G (1996) Molecular characterization of the levansucrase gene from the endophytic sugarcane bacterium Acetobacter diazotrophicus SRT4. Microbiology 142:1077-1085

Bailey MJ (1988) A note on the use of dinitrosalicylic acid for determining the products of enzymatic reactions. Appl Microbiol Biotechnol 29:494-496

Cai YP, Wolk CP (1990) Use of a conditionally lethal gene in Anabaena sp. strain PCC 7120 to select for double recombinants and to entrap insertion sequences. J Bacteriol 172:3138-3145

Chambert R, Petit-Glatron MF (1991) Polymerase and hydrolase activities of Bacillus subtilis levansucrase can be separately modulated by site-directed mutagenesis. Biochem J 279:35-41

Chowdhury B, Garai G (2017) A review on multiple sequence alignment from the perspective of genetic algorithm. Genomics 109:419-431

Dziewit L, Bartosik D (2015) Comparative analyses of extrachromosomal bacterial replicons, identification of chromids, and experimental evaluation of their indispensability. Methods Mol Biol 1231:15-29

Gao S, Qi X, Hart DJ, Gao H, An Y (2017a) Expression and characterization of Levansucrase from Clostridium acetobutylicum. J Agric Food Chem 65:867-871

Gao S, Yao S, Hart DJ, An Y (2017b) Signal peptide-dependent protein translocation pathway is crucial for the sucrose sensitivity of SacBexpressing Escherichia coli. Biochem Eng J 122:71-74

Henrissat B, Davies G (1997) Structural and sequence-based classification of glycoside hydrolases. Curr Opin Struct Biol 7:637-644

Hernandez L, Arrieta J, Menendez C, Vazquez R, Coego A, Suarez V, Selman G, Petit-Glatron MF, Chambert R (1995) Isolation and enzymic properties of levansucrase secreted by Acetobacter diazotrophicus SRT4, a bacterium associated with sugar cane. Biochem J 309:113-118

Iwabe N, Kuma K, Hasegawa M, Osawa S, Miyata T (1989) Evolutionary relationship of archaebacteria, eubacteria, and eukaryotes inferred from phylogenetic trees of duplicated genes. Proc Natl Acad Sci U S A 86:9355-9359

Jäger W, Schäfer A, Kalinowski J, Pühler A (1995) Isolation of insertion elements from gram-positive Brevibacterium, Corynebacterium and Rhodococcus strains using the Bacillus subtilis sacB gene as a positive selection marker. FEMS Microbiol Lett 126:1-6

Jäger W, Schäfer A, Pühler A, Labes G, Wohlleben W (1992) Expression of the Bacillus subtilis sacB gene leads to sucrose sensitivity in the gram-positive bacterium Corynebacterium glutamicum but not in Streptomyces lividans. J Bacteriol 174:5462-5465

Khetrapal V, Mehershahi K, Rafee S, Chen S, Lim CL, Chen SL (2015) A set of powerful negative selection systems for unmodified Enterobacteriaceae. Nucleic Acids Res 43:e83

Li W, Yu S, Zhang T, Jiang B, Mu W (2015) Recent novel applications of levansucrases. Appl Microbiol Biotechnol 99:6959-6969

Manival X, Yang Y, Strub MP, Kochoyan M, Steinmetz M, Aymerich S (1997) From genetic to structural characterization of a new class of RNA-binding domain within the SacY/BglG family of antiterminator proteins. EMBO J 16:5019-5029

Meng G, Fütterer K (2003) Structural framework of fructosyl transfer in Bacillus subtilis levansucrase. Nat Struct Biol 10:935-941

Martínez-Fleites C, Ortíz-Lombardía M, Pons T, Tarbouriech N, Taylor EJ, Arrieta JG, Hernández L, Davies GJ (2005) Crystal structure of levansucrase from the Gram-negative bacterium Gluconacetobacter diazotrophicus. Biochem J 390:19-27 
Núñez-López G, Herrera-González A, Hernández L, Amaya-Delgado L, Sandoval G, Gschaedler A, Arrizon J, Remaud-Simeon M, Morel S (2019) Fructosylation of phenolic compounds by levansucrase from Gluconacetobacter diazotrophicus. Enzym Microb Technol 122:19 25

Öner ET, Hernández L, Combie J (2016) Review of levan polysaccharide: from a century of past experiences to future prospects. Biotechnol Adv 34:827-844

Ozimek LK, Kralj S, Kaper T, Maarel MJ, Dijkhuizen L (2006) Single amino acid residue changes in subsite -1 of inulosucrase from Lactobacillus reuteri 121 strongly influence the size of products synthesized. FEBS J 273:4104-4113

Ozimek LK, van Hijum SA, van Koningsveld GA, Der Maarel van MJ, van Geel-Schutten GH, Dijkhuizen L (2004) Site-directed mutagenesis study of the three catalytic residues of the fructosyltransferases of Lactobacillus reuteri 121. FEBS Lett 560:131-133

Packer MS, Liu DR (2015) Methods for the directed evolution of proteins. Nat Rev Genet 16:379-394

Pelicic V, Reyrat JM, Gicquel B (1996) Expression of the Bacillus subtilis sacB gene confers sucrose sensitivity on mycobacteria. J Bacteriol 178:1197-1199

Polsinelli I, Caliandro R, Salomone-Stagni M, Demitri N, Rejzek M, Field RA, Benini S (2019) Comparison of the Levansucrase from the epiphyte Erwinia tasmaniensis vs its homologue from the phytopathogen Erwinia amylovora. Int J Biol Macromol 127:496-501

Porras-Domínguez JR, Rodríguez-Alegría ME, Ávila-Fernández Á, Montiel-Salgado S, López-Munguía A (2017) Levan-type fructooligosaccharides synthesis by a levansucrase-endolevanase fusion enzyme (LevB1SacB). Carbohydr Polym 177:40-48

Rubio MÁ, Napolitano M, Ochoa de Alda JA, Santamaría-Gómez J, Patterson CJ, Foster AW, Bru-Martínez R, Robinson NJ, Luque I (2015) Trans-oligomerization of duplicated aminoacyl-tRNA synthetases maintains genetic code fidelity under stress. Nucleic Acids Res 43:9905-9917

Sawisit A, Jantama K, Zheng H, Yomano LP, York SW, Shanmugam KT, Ingram LO (2015) Mutation in galP improved fermentation of mixed sugars to succinate using engineered Escherichia coli AS1600a and AM1 mineral salts medium. Bioresour Technol 193: 433-441

Seo JW, Song KB, Jang KH, Kim CH, Jung BH, Rhee SK (2000) Molecular cloning of a gene encoding the thermoactive levansucrase from Rrahnella aquatilis and its growth phase-dependent expression in Eescherichia coli. J Biotechnol 81:63-72
Shimamura A, Tsuboi K, Nagase T, Ito M, Tsumori H, Mukasa H (1987) Structural determination of D-fructans from Streptococcus mutans, serotype b, c, e, and f strains, by 13C-n.m.r. spectroscopy. Carbohydr Res 165:150-154

Strube CP, Homann A, Gamer M, Jahn D, Seibel J, Heinz DW (2011) Polysaccharide synthesis of the levansucrase SacB from Bacillus megaterium is controlled by distinct surface motifs. J Biol Chem 286:17593-17600

Szwengiel A, Wiesner M (2019) Effect of metal ions on levan synthesis efficiency and its parameters by levansucrase from Bacillus subtilis. Int J Biol Macromol 128:237-243

Thulin E, Sundqvist M, Andersson DI (2015) Amdinocillin (Mecillinam) resistance mutations in clinical isolates and laboratory-selected mutants of Escherichia coli. Antimicrob Agents Chemother 59:17181727

Verhaest M, Van den Ende W, Roy KL, De Ranter CJ, Laere AV, Rabijns A (2005) X-ray diffraction structure of a plant glycosyl hydrolase family 32 protein: fructan 1-exohydrolase IIa of Cichorium intybus. Plant J 41:400-411

Weissman KJ (2016) Genetic engineering of modular PKSs: from combinatorial biosynthesis to synthetic biology. Nat Prod Rep 33:203230

Wuerges J, Caputi L, Cianci M, Boivin S, Meijers R, Benini S (2015) The crystal structure of Erwinia amylovora levansucrase provides a snapshot of the products of sucrose hydrolysis trapped into the active site. J Struct Biol 191:290-298

Xu W, Ni D, Zhang W, Guang C, Zhang T, Mu W (2018) Recent advances in levansucrase and inulosucrase: evolution, characteristics, and application. Crit Rev Food Sci Nutr 30:1-18

Xu W, Yu S, Liu Q, Zhang T, Jiang B, Mu W (2017) Enzymatic production of melibiose from raffinose by the levansucrase from Leuconostoc mesenteroides B-512 FMC. J Agric Food Chem 65: 3910-3918

Zaremba-Niedzwiedzka K, Caceres EF, Saw JH, Bäckström D, Juzokaite L, Vancaester E, Seitz KW, Anantharaman K, Starnawski P, Kjeldsen KU, Stott MB, Nunoura T, Banfield JF, Schramm A, Baker BJ, Spang A, Ettema TJ (2017) Asgard archaea illuminate the origin of eukaryotic cellular complexity. Nature 541:353-358

Publisher's note Springer Nature remains neutral with regard to jurisdictional claims in published maps and institutional affiliations. 